\title{
Hypoxia-preconditioned mesenchymal stem cells attenuate microglial pyroptosis after intracerebral hemorrhage
}

\author{
Jianyang Liu ${ }^{1}$, Jialin $\mathrm{He}^{1}$, Yan Huang ${ }^{2}$, Lite Ge ${ }^{1}$, Han Xiao ${ }^{1}$, Liuwang Zeng ${ }^{1}$, Zheng Jiang ${ }^{1}$, Ming Lu ${ }^{3,4}$, \\ Zhiping $\mathbf{H u}^{1}$
}

${ }^{1}$ Department of Neurology, Second Xiangya Hospital, Central South University, Changsha, China; ${ }^{2}$ National Health Commission Key Laboratory of Birth Defects Research, Prevention, and Treatment, Hunan Provincial Maternal and Child Health Care Hospital, Changsha, China; ${ }^{3}$ Developmental Biology of Ministry of Education, College of Life Sciences, Hunan Normal University, Changsha, China; ${ }^{4}$ Hunan Provincial Key Laboratory of Neurorestoratology, Second Affiliated Hospital of Hunan Normal University, Changsha, China

Contributions: (I) Conception and design: Z Hu, M Lu; (II) Administrative support: J Liu; (III) Provision of study materials or patients: Y Huang, L Ge, H Xiao, L Zeng; (IV) Collection and assembly of data: J Liu, J He, Z Jiang; (V) Data analysis and interpretation: J Liu; (VI) Manuscript writing: All authors; (VII) Final approval of manuscript: All authors.

Correspondence to: Zhiping Hu. Department of Neurology, Second Xiangya Hospital, Central South University, Changsha, China. Email: zhipinghu@csu.edu.cn. Ming Lu. Developmental Biology of Ministry of Education, College of Life Sciences, Hunan Normal University, Changsha, China. Email: lumingcs163@163.com.

Background: Microglia plays a vital role in neuroinflammation, contributing to the pathogenesis of intracerebral hemorrhage (ICH)-induced brain injury. Mesenchymal stem cells (MSCs) hold great potential for treating ICH. We previously revealed that MSCs ameliorate the microglial pyroptosis caused by an ischemic stroke. However, whether MSCs can modulate microglial pyroptosis after ICH remains unknown. This study aimed to investigate the neuroprotective effects of hypoxia-preconditioned olfactory mucosa MSCs (OM-MSCs) on ICH and the possible mechanisms.

Methods: ICH was induced in mice via administration of collagenase IV. At $6 \mathrm{~h}$ post-ICH, $2-4 \times 10^{5}$ normoxic/hypoxic OM-MSCs or saline were intracerebrally administered. To evaluate the neuroprotective effects, the behavioral outcome, apoptosis, and neuronal injury were measured. Microglia activation and proinflammatory cytokines were applied to detect neuroinflammation. Microglial pyroptosis was determined by western blotting, immunofluorescence staining, and transmission electron microscopy (TEM).

Results: The two OM-MSC-transplanted groups exhibited significantly improved functional recovery and reduced neuronal injury, especially the hypoxic OM-MSCs group. Hypoxic OM-MSCs attenuated microglial activation as well as the levels of interleukin-1 $\beta$ (IL-1 $\beta$ ) and tumor necrosis factor- $\alpha$ (TNF- $\alpha$ ). Moreover, we found that hypoxia-preconditioned OM-MSCs ameliorated pyroptosis by diminishing the levels of pyroptosis-associated proteins in peri-hematoma brain tissues, decreasing the expression of the microglial nod-like receptor family protein 3 (NLRP3) and caspase-1, and reducing the membrane pores on microglia post-ICH.

Conclusions: Our study showed that hypoxic preconditioning augments the therapeutic efficacy of OMMSCs, and hypoxia-preconditioned OM-MSCs alleviate microglial pyroptosis in the ICH model.

Keywords: Mesenchymal stem cells (MSCs); intracerebral hemorrhage (ICH); hypoxic preconditioning; microglia; pyroptosis

Submitted May 19, 2021. Accepted for publication Jul 14, 2021.

doi: 10.21037/atm-21-2590

View this article at: https://dx.doi.org/10.21037/atm-21-2590 


\section{Introduction}

Intracerebral hemorrhage (ICH) causes $15-20 \%$ of all strokes and results in higher rates of mortality and disability in the population compared with ischemic stroke (1). After ICH, secondary brain injury, which mainly involves neuroinflammatory reactions, is an essential contributor to the high disability rate post-ICH (2). Previous studies have demonstrated that the inflammatory reaction runs over the entire course of ICH in the animal model (3). Cumulative inflammatory responses promote the development of brain injury and neurological deficits (4). Microglia, the critical immune cells in the central nervous system, is a vital source of the neuroinflammatory response after $\operatorname{ICH}(5,6)$. Under stroke conditions, microglia become activated and release inflammasome components and pro-inflammatory cytokines. In an environment of microbial products, the M1 phenotype is activated. The secretion of pro-inflammatory cytokines characterizes M1-like microglia. However, microglia may assume the M2 phenotype, which expresses anti-inflammatory cytokines. Thus, microglia play a dual role in different pathological stages after $\mathrm{ICH}(7,8)$.

Previous studies have suggested that regulating microglia polarization is a potentially effective strategy for ICH treatment. Repolarizing microglia towards the M2 phenotype contributes to promoting functional nerve recovery in the $\mathrm{ICH}$ model (9-11). The mechanism regulating microglia polarization after $\mathrm{ICH}$ involves various signaling pathways, such as the Dectin-1/spleen tyrosine kinase/caspase recruitment domain-containing protein 9/ nuclear factor-kappa B (12), mitochondrial ROS/nodlike receptor family protein 3 (NLRP3) (13), cannabinoid receptor-2/phosphorylated cAMP-dependent protein kinase/cAMP-response element binding protein (14), Janus kinase $1 /$ transcription $6(15)$, peroxisome proliferatoractivated receptor gamma/cluster of differentiation 36 (16), and programmed death protein 1/programmed deathligand 1 pathway (17). Driving microglia towards an M2 phenotype could restrict microglia-mediated inflammatory responses. Although some research has been focused on elaborating the internal mechanisms of microglial polarization, there are still other mechanisms involved in microglia-mediated inflammatory responses after ICH that have not yet been elucidated.

Pyroptosis, a programmed form of death, is characterized by cell swelling, membrane rupture, pore formation, DNA damage, and the release of pro-inflammatory cellular contents (18) and can be triggered by ICH $(19,20)$. The effector protein, Gasdermin D (GSDMD), is the executor of pyroptosis (21). After being cleaved by activated caspase-1, the mobilized N-domain of GSDMD forms membrane pores. Meanwhile, caspase- 1 cleaves the inactive prointerleukin (IL)-18 and pro-IL-1 $\beta$ into mature IL-18 and IL-1 $\beta$ (22), which can be released through the membrane pores, thereby perpetuating the inflammation cascade (23). Caspase- 1 activation is mediated by inflammasomes, which are multimeric complexes (24). The most studied inflammasome, NLRP3, has been shown in the animal model of ICH $(13,19,25,26)$. NLRP3 is mainly expressed in brain microglia and significantly increases at 3 hours, peaks at 12 hours, and remains at high levels at 72 hours after ICH (19). Ischemic (27-29) or hemorrhagic stroke (20) can induce apoptotic and pyroptotic microglial cell death. Suppressing microglial pyroptosis alleviates brain-blood barrier disruption and neuronal injury after subarachnoid hemorrhage (30), and improves survival and neurological outcomes after cardiac arrest (31).

Cell-based therapy is a promising avenue of $\mathrm{ICH}$ cure $(32,33)$. Various sources of mesenchymal stem cells (MSCs) have been applied in ICH, such as adipose $(34,35)$, bone marrow (36), umbilical cord (37), and umbilical cord blood (38). A previous meta-analysis suggested that stem cell therapy showed significant behavioral outcome improvements in the ICH animal model (39). Recently, hypoxic preconditioning was reported to enhance the therapeutic efficacy of MSCs (40-43). Hypoxiapreconditioned ( $0.1-0.3 \%$ oxygen concentration) bone marrow-derived MSCs have been shown to stimulate neurogenesis after ICH (44) dramatically. Also, 5\% hypoxic preconditioning was demonstrated to increase vascular endothelial growth factor secretion and enhance the tissueprotective capability of neural stem cells in ICH (45).

Olfactory mucosa MSCs (OM-MSCs) localized in the nasal lamina propria have recently been recognized as an ideal source of MSCs, as they are easily accessible and situated for autologous transplantation (46). Previously, we demonstrated that OM-MSCs exerts neuroprotective effects in ischemic stroke $(47,48)$. We also revealed that hypoxia-preconditioned OM-MSCs inhibited microglial pyroptosis in an in vitro model (49) and affirmed that hypoxic preconditioning enhanced the therapeutic efficacy of OM-MSCs in an in vivo model (50). However, to our knowledge, there are no studies that have explored the antiinflammatory properties of hypoxia-preconditioned OMMSC therapy in an in vivo model of ICH. In this study, we intended to explore the effect of hypoxic preconditioning 
on the therapeutic efficacy of OM-MSCs in treating ICH. We further clarified the possible mechanism through which hypoxic preconditioning alleviates inflammation injury after ICH. Collectively, our study aims to demonstrate a strategy to improve the efficacy of OM-MSCs in ICH and elaborate on the possible mechanism. We present the following article in accordance with the ARRIVE reporting checklist (available at https://dx.doi.org/10.21037/atm-21-2590).

\section{Methods}

\section{Isolation of OM-MSCs}

Human OM-MSCs from healthy adult volunteers (two males, two females, 20-40 years old) were obtained from the surface interior of the concha nasalis media during otolaryngology endoscopy surgery. Informed consent was obtained from each subject preoperatively. The study was conducted in accordance with the Declaration of Helsinki (as revised in 2013), and the procedure protocol was approved by the ethics committee of Hunan Normal University (No. 2009163009). The olfactory mucosa was isolated according to a published protocol (46). The third passage of OM-MSCs was selected for use in this experiment. The cells derived from the two different sexes were randomly administrated into different mice.

\section{Hypoxic preconditioning}

The OM-MSCs were incubated at $37{ }^{\circ} \mathrm{C}$ and $3 \% \mathrm{O}_{2}, 5 \%$ $\mathrm{CO}_{2}$, and $92 \% \mathrm{~N}_{2}$ for 48 hours in a gas-tight humidified chamber (modular incubator chamber; Billups-Rothenberg, Del Mar, CA, USA).

\section{Animals}

All male C57BLC/6 healthy mice (weighing 25-28 g) were housed in the animal center of Hunan Normal University, with controlled temperature and light cycles conditions (12 h light/dark cycles, and $20-25^{\circ} \mathrm{C}$ ). Experiments were performed under a project license (No. 2020-110 164) granted by the institutional ethics board of Hunan Normal University, in compliance with the National Institutes of Health Guide for Care and Use of Laboratory Animals.

\section{ICH model in vivo}

All mice $(\mathrm{n}=90)$ were randomized into a sham-operated group ( $\mathrm{n}=20), \mathrm{ICH}+$ saline group, $\mathrm{ICH}+$ normoxic OMMSCs group, and $\mathrm{ICH}+$ hypoxic OM-MSCs group. The mice were anesthetized with $3.5 \%$ isoflurane and maintained with $2.0 \%$ isoflurane in $30 \% \mathrm{O}_{2}$ and $70 \%$ $\mathrm{N}_{2} \mathrm{O}$ mixture. The injection of collagenase IV successfully induced ICH ( $0.075 \mathrm{U}$ dissolved in $1.0 \mu \mathrm{L}$ saline; position: $0.2 \mathrm{~mm}$ anterior, $2.3 \mathrm{~mm}$ lateral, and $3.6 \mathrm{~mm}$ ventral bregma at a rate of $0.1 \mu \mathrm{L} / \mathrm{min}$ ) (Sigma-Aldrich, St. Louis, USA). The needle was slowly removed after 10 minutes. The mice were placed on a heating pad to maintain their body temperature at $37.0{ }^{\circ} \mathrm{C}$ during the period of modeling and recovery from anesthesia. The sham-operated group underwent the same procedures without the injection of collagenase IV.

\section{OM-MSCs intracerebral transplantation}

After surgery, the surviving animals were randomized into a normoxic $(n=20)$ or hypoxic $(n=20)$ MSCs group, or saline group $(\mathrm{n}=20)$, at $6 \mathrm{~h}$ post-ICH surgery (Figure 1A). For the $\mathrm{ICH}+$ MSCs treated group, $2-4 \times 10^{5}$ OM-MSCs in $2 \mu \mathrm{L}$ saline were stereotactically transplanted into the edge of the ipsilateral lesion area. For the $\mathrm{ICH}+$ saline-treated group, $2 \mu \mathrm{L}$ saline was administrated in the same position.

\section{Neurobehavioral tests}

A standardized battery of behavioral tests was used to quantify neurological function before and at 3 days, 7 days, and 14 days after ICH with different treatments. The modified neurological severity scores (mNSS) (51) and rotarod test were blindly performed to assess a neurological deficiency. The speed was slowly increased from 4 to $40 \mathrm{rpm}$ for rotarod tests over a $5 \mathrm{~min}$ period for rotarod tests. The mice performed rotarod training for 3 days before ICH.

\section{Tissue preparation}

The mice were transcardially perfused with $0.9 \%$ ice-cold saline, followed by $4 \%$ paraformaldehyde in phosphate buffered saline (PBS). After perfusion, the whole brains were removed, kept in the same fixative for $4 \mathrm{~h}$ at $4{ }^{\circ} \mathrm{C}$, and immersed in $20 \%$ sucrose in $0.1 \mathrm{M}$ phosphate buffer solution until saturated. $10 \mu \mathrm{m}$ coronal sections were cut on a cryostat (Leica CM1850, Wetzlar, Germany) between +4.7 to $-5.2 \mathrm{~mm}$ from bregma and stored at $-20{ }^{\circ} \mathrm{C}$ for subsequent staining. 

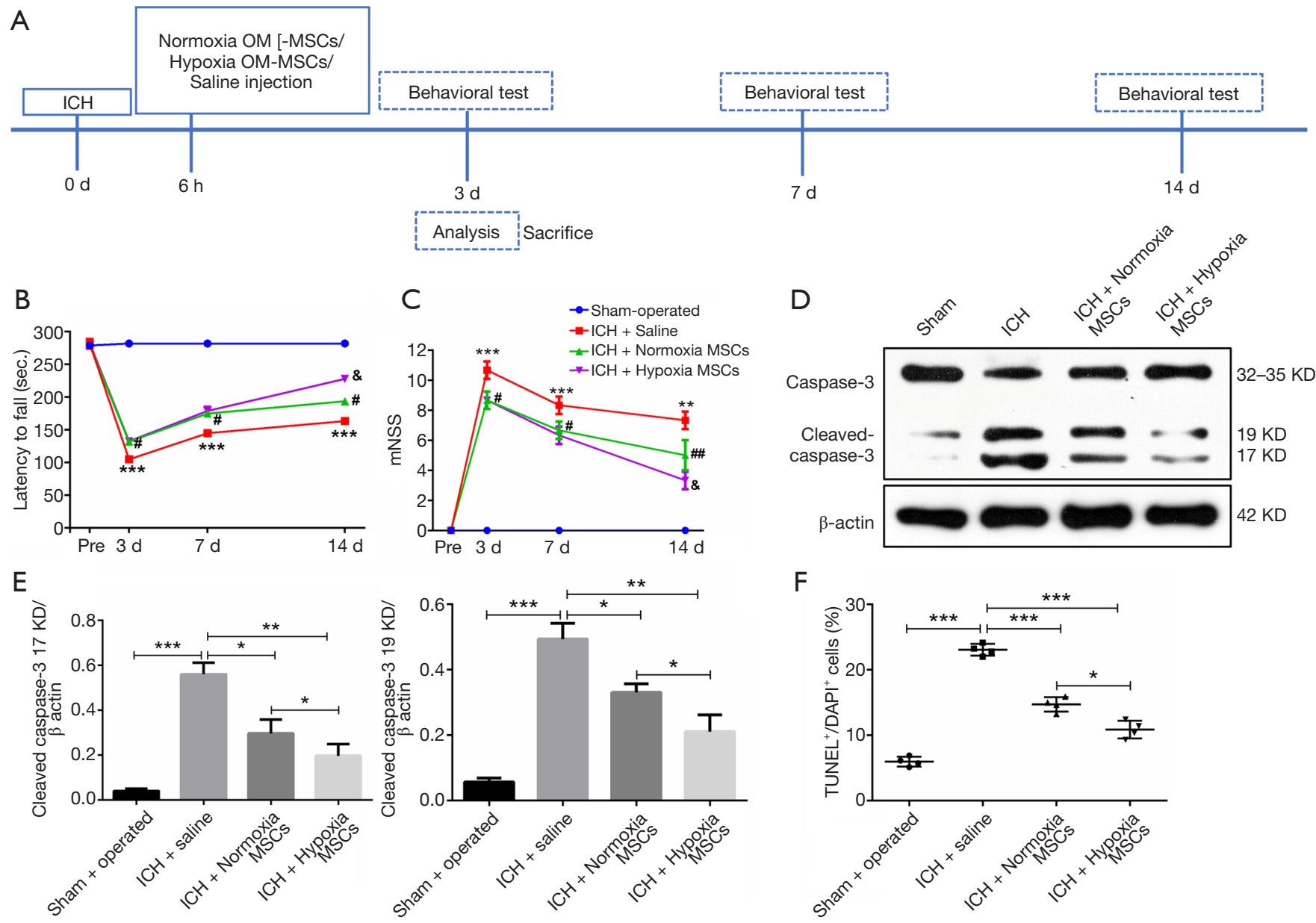

F
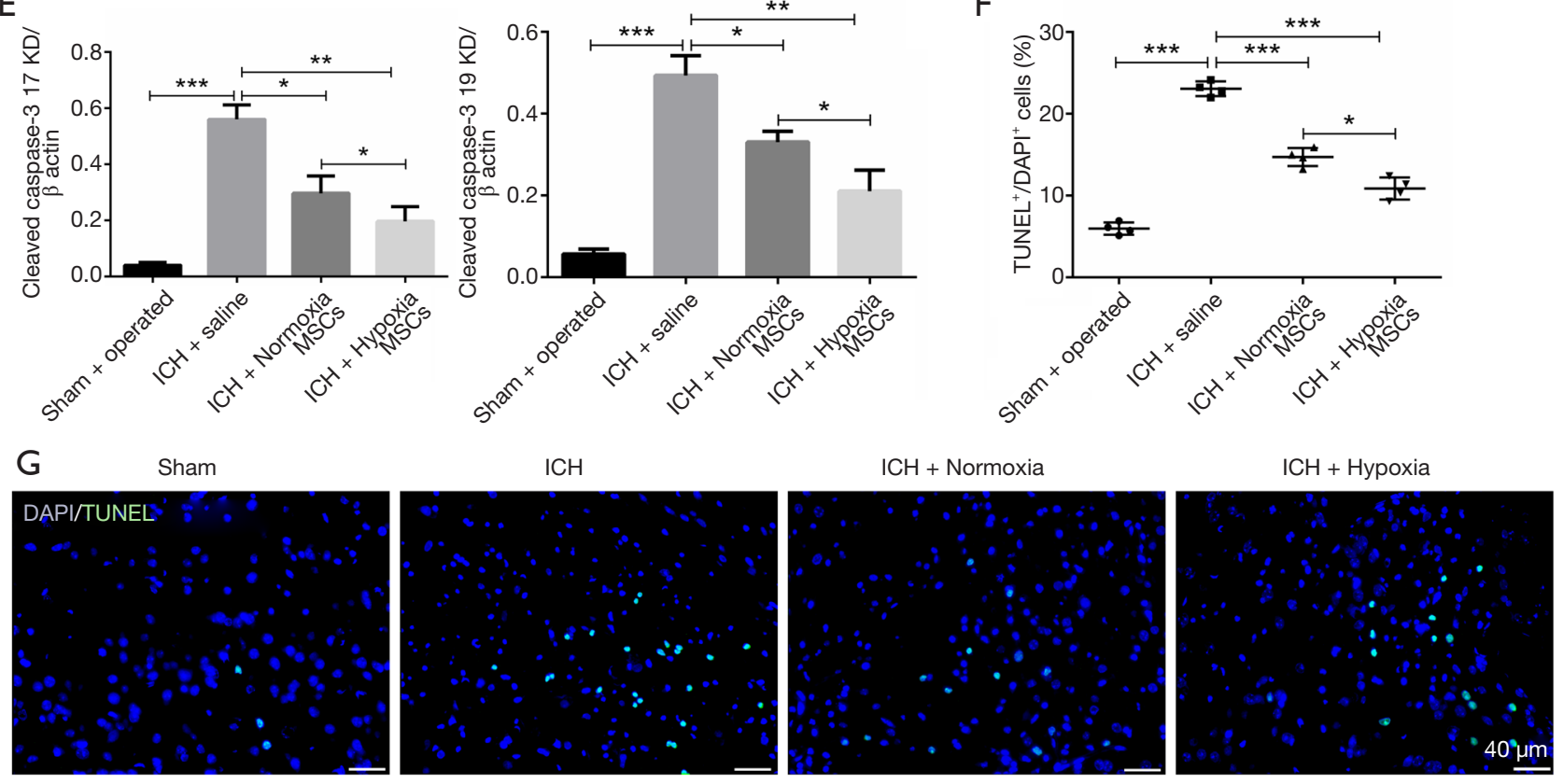

Figure 1 OM-MSCs alleviated the neurological deficit and cell apoptosis in the in vivo model of ICH. (A) Schematic representation of the experimental protocol. (B) The rotarod test, and (C) the mNSS test were assessed before ICH and on day 3 , 7, and 14 post-ICH. Data were expressed as the mean $\pm \mathrm{SEM}$ ( $\mathrm{n}=7$ /group at 3 days; $\mathrm{n}=5$ /group at 7 and 14 days) ${ }^{* * *}, \mathrm{P}<0.001,{ }^{* *}, \mathrm{P}<0.01$ vs. sham-operated; \#, $\mathrm{P}<0.05$, \#\#, $\mathrm{P}<0.01$ vs. ICH + saline; ${ }^{\&}, \mathrm{P}<0.05$ vs. ICH + Normoxic MSCs). (D,E) Western blot images and densitometric analysis of caspase-3. (F) Quantification of TUNEL staining was expressed as (TUNEL-stained nuclei/DAPI-stained nuclei) $\times 100 \%$. Sections were stained with DAPI (blue) to show all nuclei. TUNEL-positive cells were increased at 3 days after ICH, and were decreased by OM-MSC administration, especially hypoxic OM-MSCs. Data were expressed as the mean \pm SEM $(\mathrm{n}=4)\left({ }^{*}, \mathrm{P}<0.05 ;{ }^{* *}, \mathrm{P}<0.01 ;{ }^{* * *}, \mathrm{P}<0.001\right)$. $(\mathrm{G})$ Representative images of TUNEL staining from the ipsilateral cortex of the perihematomal area. The scale bar is $40 \mu \mathrm{m}$. Abbreviations: OM-MSCs, olfactory mucosa mesenchymal stem cells; ICH, intracerebral hemorrhage; mNSS, modified neurological severity score; SEM, standard error of mean; TUNEL, terminal deoxynucleotidyl transferase biotin-mediated dUTP Nick-end labeling; DAPI, 6-diamidino-2phenylindole. 


\section{TUNEL assay}

Terminal deoxynucleotidyl transferase biotin-mediated dUTP Nick-end labeling (TUNEL) staining was performed according to the manufacturer's instructions (Promega Corporation, Madison, WI, United States). Nuclei were stained with 6-diamidino-2-phenylindole (DAPI; Sigma; USA). For each coverslip, five random fields in the cortex of the perihematomal region were examined under a fluorescent microscope. The total number of TUNEL positive cells were counted using ImageJ software (National Institute of Health [NIH], Bethesda, MD, USA). The result was presented as the ratio between the number of TUNEL positive cells, and the number of DAPI-stained nuclei counted $\times 100 \%$.

\section{Nissl staining}

Brain paraffin sections were placed in $0.2 \%$ Nissl staining solution for $5 \mathrm{~min}$ at room temperature. Representative images of Nissl-stained brain sections in the cortex of the perihematomal region on day 3 post-ICH were captured under a high-power light microscope (Olympus, Tokyo, Japan). The number of apoptotic neurons was counted using ImageJ software (NIH, USA).

\section{Hematoxylin and eosin (HE) staining}

Sections were cut from paraffin blocks and stained with HE. According to the manufacturer's directions, HE staining was performed with a staining kit (Solarbio, Beijing, China). Representative images of HE-stained brain sections in the cortex of the perihematomal region on day 3 following ICH were captured under a high-power light microscope (Olympus, Tokyo, Japan).

\section{Immunofluorescence staining}

Perihematomal brain sections were washed with phosphate buffer solution tween (PBST) three times for $3 \mathrm{~min}$ each and then blocked with a solution containing $0.3 \%$ Triton, 3\% goat serum, and 1\% bovine Serum Albumin (BSA) for $1 \mathrm{~h}$. Subsequently, sections were incubated with primary antibodies including: anti-Iba-1 (1:500; $10904-$ 1-AP; Proteintech; USA), anti-caspase-1 (1:100; 22915 1-AP; Proteintech), and anti-NLPR3 (1:200; PA5-88709; Thermo Fisher Scientific; USA) overnight at $4{ }^{\circ} \mathrm{C}$. After being washed with PBS three times for $3 \mathrm{~min}$, the slices were incubated with corresponding fluorescence-conjugated secondary antibodies (1:500, Proteintech) for $2 \mathrm{~h}$, followed by staining with DAPI for $10 \mathrm{~min}$. The positive cells were analyzed by an observer blinded to groups using Image $\mathrm{J}$ software (NIH, USA).

\section{Western blot analysis}

Proteins were extracted from the perihematomal brain tissue using a total protein extraction kit (Beyotime, China). Protein concentrations were examined by a bicinchoninic acid (BCA) protein assay kit (Beyotime, China). Protein extracts were separated by sodium dodecyl sulfate polyacrylamide gel electrophoresis (SDS-PAGE) and then immunoblotted. The following primary antibodies were applied: caspase3 (1: 1,000; 19677-1-AP; Proteintech; USA), NLRP3 (1:1,000; \#15101; CST; USA), apoptosis-associated speck-like proteins containing a caspase recruitment domain (ASC) $(1: 1,000 ; 67494-1-\mathrm{Ig}$; Proteintech; USA), caspase-1 (1:1,000; ab179515; Abcam; Cambridge), caspase8 (1:750; 13423-1-AP; Proteintech; USA), IL- $1 \beta(0.15 \mu \mathrm{g} / \mathrm{mL}$; ab9722; Abcam), GSDMD (1:1,000; ab219800; Abcam), glyceraldehyde-3-phosphate dehydrogenase (GAPDH) (1:5,000; 10494-1-AP; Proteintech; USA) at $4{ }^{\circ} \mathrm{C}$ overnight. Secondary antibodies included horseradish peroxidaseconjugated anti-mouse or anti-rabbit immunoglobulin G (IgG) (1:5,000; SA00001-1; SA00001-2; Proteintech). Finally, the proteins were visualized using enhanced chemiluminescence substrate (Bio-Rad, Munich, Germany).

\section{Real-time polymerase chain reaction (RT-PCR)}

Total ribonucleic acid (RNA) was obtained from the perihematomal tissues using Trizol reagent (Invitrogen, Carlsbad, CA, USA). The transcript amount of the $\beta$-actin housekeeping gene was used as an internal RNA control. The TNF- $\alpha$ forward: 5 '-CA CGCTCT TC TG TCT ACTGA A C-3' and reverse: 5'- AT CT GAG TG TGA GGG T C T GG-3'; IL-1 $\beta$ forward: 5 '-CAG GCAA CCA CT TA CCTATTTA-3' and reverse: 5'-CCATA CAC ACGGACAA CAA CTA GA T -3'; $\beta$-actin forward: 5'-GG CATCGTGATGGACTCCG-3' and reverse: 5'-GCTGGAAGGTGGACAGCGA -3'. The threshold cycle value (CT) was calculated by the $\Delta \Delta \mathrm{CT}$ method.

\section{Transmission electron microscopy (TEM)}

The perihematomal regions from the fresh brain samples were separated and embedded in epoxy resin. Observations 
were carried out using an electron microscope (Hitachi, HT7700, Japan).

\section{Statistical analysis}

The results were expressed as the mean \pm standard error of mean (SEM). Comparisons between groups were estimated using the two-sided unpaired Student's $t$-test or twosided Analysis of Variance (ANOVA), with the Bonferroni correction used for the post hoc $t$-test as appropriate. Statistical analysis was calculated using GraphPad Prism 6 Software (GraphPad Software, Inc., San Diego, CA, USA). $P$ values $<0.05$ were considered significant.

\section{Results}

\section{OM-MSCs ameliorated neurological deficit after ICH}

Our previous studies have verified the cellular morphology and expression profiles of surface antigens on OM-MSCs (47-49,52). OM-MSCs were stereotactically transplanted into the surroundings of the bleeding area at 6 hours post$\mathrm{ICH}$. To assess the neuroprotective effects of normoxic and hypoxic OM-MSCs, a series of tests were evaluated at 3, 7, and 14 days after ICH (Figure 1A).

The mNSS and rotarod test were used to evaluate the therapeutic effects of OM-MSCs on ICH. The MSCs groups, particularly the hypoxic MSCs group, showed significant improvements in the rotarod test (Figure 1B) and mNSS (Figure 1C) compared with the saline group at 14 days after ICH $(\mathrm{P}<0.05)$. There were no significant differences between the normoxic and hypoxic MSCs groups at 3 and 7 days after $\mathrm{ICH}$.

\section{Hypoxia-preconditioned OM-MSCs diminished cell apoptosis after $\mathrm{ICH}$}

Although there were no significant differences in the neurological deficit score between the normoxic and hypoxic MSCs groups at 3 days after ICH, the histological and pathological changes may differ between the groups. To determine the apoptosis in the cortex, the expression of cleaved caspase- 3 was assayed by western blot. As indicated in Figure $1 D, 1 E$, cleaved caspase-3 was increased after exposure to ICH but partially reversed by OM-MSCs treatment, especially hypoxic OM-MSCs $(\mathrm{P}<0.05)$. In addition, TUNEL staining was performed 3 days after ICH. The administration of OM-MSCs significantly decreased the number of $\mathrm{TUNEL}^{+}$cells compared to that in the saline group. Moreover, hypoxic preconditioning enhanced this protective effect at 3 days after ICH (Figure $1 F, 1 G$, $\mathrm{P}<0.05)$. These tests collectively suggested that hypoxiapreconditioned OM-MSCs increased neuron survival and reduced cleaved caspase- 3 maturation after $\mathrm{ICH}$.

\section{Hypoxic preconditioning enbanced the neuroprotection of OM-MSCs in the ICH model}

To investigate the neuronal survival after ICH, Nissl staining was performed 3 days after ICH. Figure $2 A$ shows the Nissl staining images, which reveal neuronal density in the brain with or without treatment with normoxic or hypoxic OM-MSCs at 3 days post-ICH. The injured neurons were characterized by small, shrunken, and dark nuclei, while the normal neurons had vesicular nuclei with centrally located nucleoli. As revealed in Figure 2B, few neuronal deaths occurred in the sham-operated group. In the ICH + saline group, extensive neuronal loss was found in the cortex. A decreased number of apoptotic neurons were observed in the brain that received OM-MSCs administration, especially in the hypoxic OM-MSCs group $(\mathrm{P}<0.01)$. Moreover, we applied the HE staining to assess the histopathological changes in the cortex. The hypoxic OM-MSCs group showed decreased eosinophilic cells compared with the normoxic OM-MSCs group at 3 days after ICH (Figure $2 C, 2 D, \mathrm{P}<0.05$ ).

Generally, both normoxic and hypoxic OM-MSCs reduced the severity of the cerebral injury. Compared to the normoxic OM-MSCs, hypoxic OM-MSC treatment significantly increased neuronal survival at 3 days post-ICH. The treatment order in terms of neuroprotection was shamoperated > hypoxic OM-MSCs administration > normoxic OM-MSCs administration > ICH + saline group.

\section{Hypoxic OM-MSCs repressed microglia activation and inflammatory cytokines after ICH}

Given the essential role of inflammatory responses in the second brain injury after ICH, we speculated that the enhanced neuroprotection of hypoxia-preconditioned OMMSCs might be related to the anti-inflammatory effects. Thus, in the following study, we determined whether hypoxia-preconditioned OM-MSCs could regulate postICH neuroinflammation. Iba-1 staining was used to analyze microglial activation in the perihematomal region. Compared with the sham-operated group, the Iba-1 positive cells 


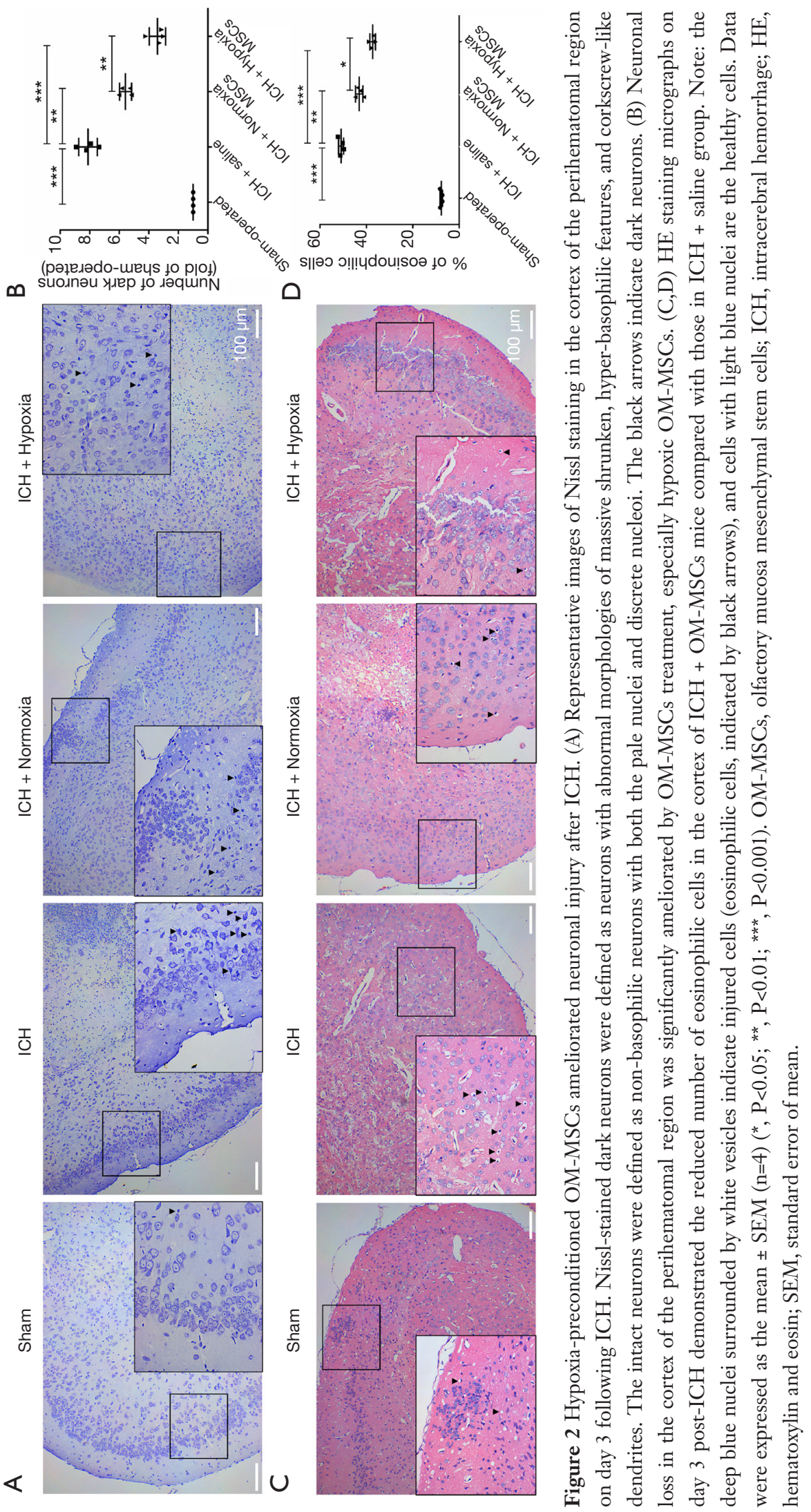



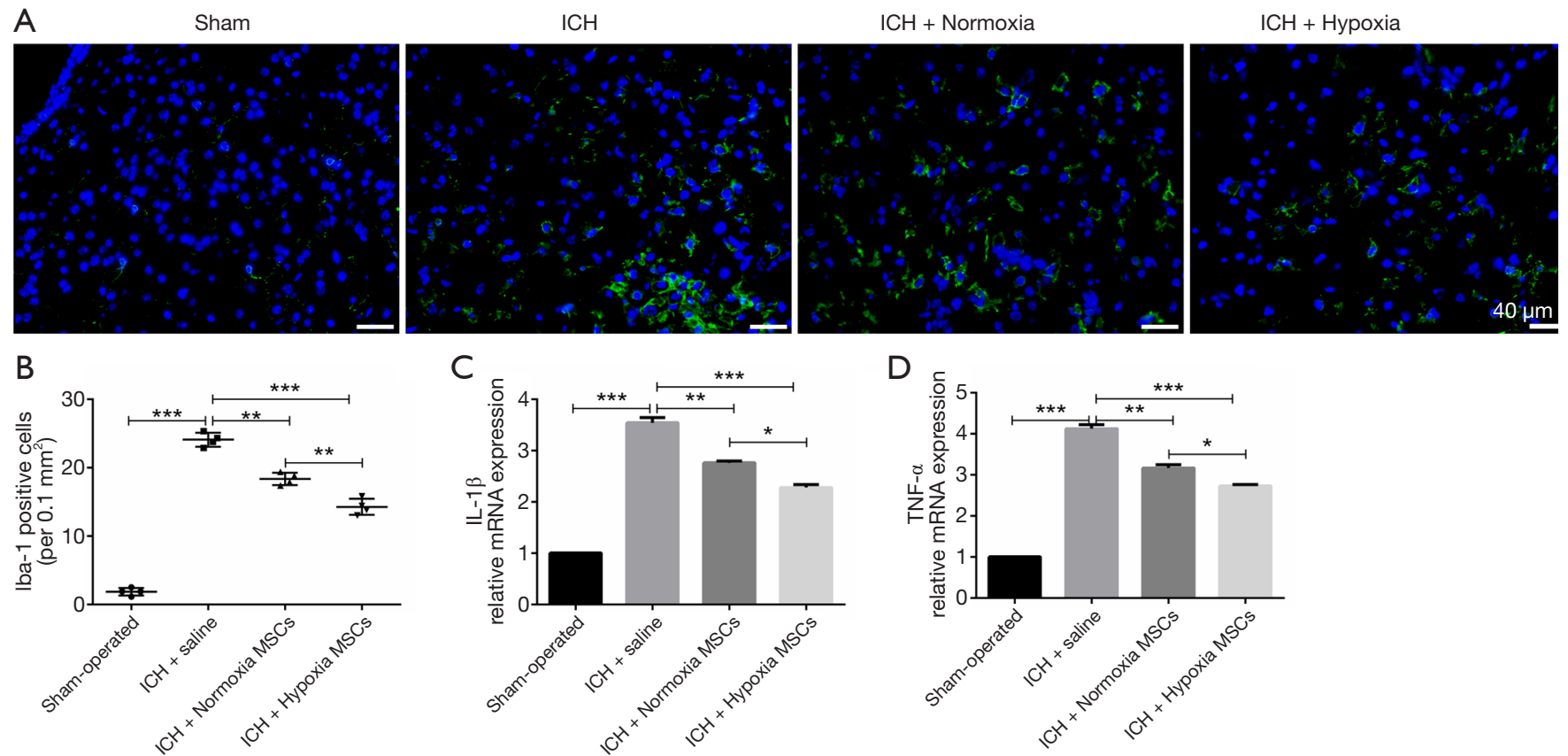

Figure 3 Hypoxia-preconditioned OM-MSCs suppressed microglial activation and inflammatory mediators. (A) Images of Iba-1 immunostaining in the ipsilateral cortex of the perihematomal region at 3 days after ICH. (B) Quantitative analysis of Iba-1 positive cells. (C) IL-1 $\beta$ production in the perihematomal regions of treated groups at 3 days post-ICH was detected by real-time PCR. (D) TNF- $\alpha$ production in the perihematomal regions of treated groups at 3 days post-ICH was detected by real-time PCR. Data were expressed as the mean $\pm \operatorname{SEM}(\mathrm{n}=4)$ (, $\mathrm{P}<0.05$; **, $\mathrm{P}<0.01$; $\left.{ }^{* *}, \mathrm{P}<0.001\right)$. Abbreviations: OM-MSCs, olfactory mucosa mesenchymal stem cells; ICH, intracerebral hemorrhage; PCR, polymerase chain reaction; SEM, standard error of mean.

significantly increased in the $\mathrm{ICH}+$ saline group. Meanwhile, fewer activated microglia were observed in OM-MSC transplanted mice, especially the hypoxia-preconditioned OM-MSCs treated group (Figure $3 A, 3 B, \mathrm{P}<0.01$ ). In addition, inflammation-associated molecules, including IL-1 $\beta$ and TNF- $\alpha$, were examined by RT-PCR at 3 days after ICH. As shown in Figure 3C,3D, the expressions of IL- $1 \beta$ and TNF- $\alpha$ were higher in the ICH group cortex than that in the sham-operated group. Compared with the ICH + saline group, the pro-inflammatory cytokine contents were lower in the ICH + OM-MSCs group $(\mathrm{P}<0.05)$.

\section{Hypoxia-preconditioned OM-MSCs alleviated microglial pyroptosis after ICH}

Pyroptosis is a vital contributor exacerbating post-ICH neuroinflammation. The pyroptosis-related molecules at 3 days post-ICH were explored to investigate the protective effects of OM-MSCs. Accordingly, ICH was found to significantly increase the levels of NLRP3, apoptosis-associated speck-like protein containing a caspase recruitment domain (ASC), cleaved caspase-1, cleaved caspase-8, GSDMD, and IL-1 $\beta$. However, these levels were abrogated by OM-MSC treatment. Compared with normoxic OM-MSCs treatment, hypoxic OM-MSCs treatment significantly attenuated the expression of cleaved caspase-8, GSDMD, and IL-1 $\beta$. (Figure 4A,4B).

We subsequently evaluated microglial pyroptosis following ICH. Iba1/NLRP3 double immunofluorescent labeling indicated that more NLRP3 positive microglial cells were observed in the ipsilateral cortex 3 days post-ICH compared with the sham-operated group, which was ameliorated by OM-MSCs administration (Figure $5 A, 5 B, \mathrm{P}<0.05$ ). The same results were presented in the Iba1/caspase-1 double immunofluorescence (Figure 5C,5D, $\mathrm{P}<0.05$ ).

Consistently, we also examined ultrastructural changes of microglia 3 days post-ICH with TEM. We observed bubbles and large holes in the plasma membrane of microglia from the ICH group, in contrast to the linear and intact membrane from the sham-operated group. The membrane pores were less frequent in the OM-MSCs treated group (Figure 5E). Taken together, these findings suggested that 


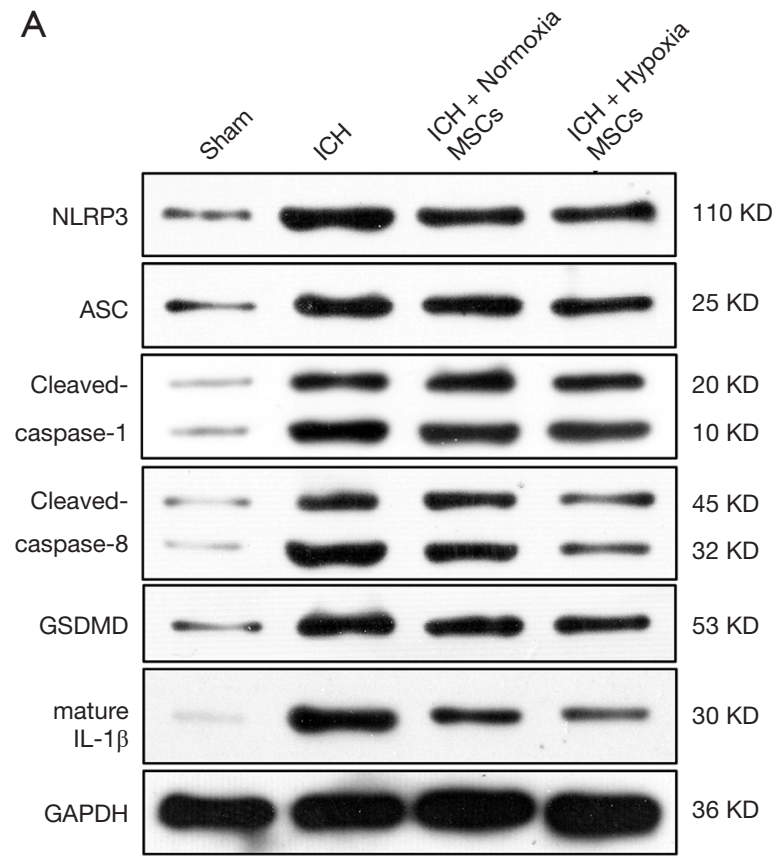

B
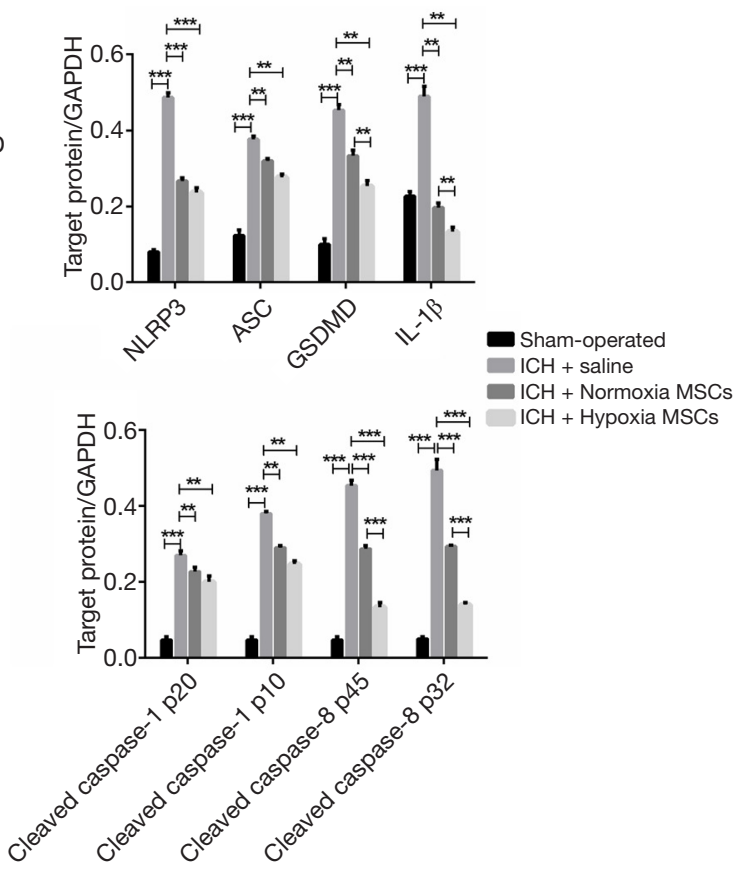

Figure 4 Hypoxia-preconditioned OM-MSCs downregulated the level of molecules closely related to pyroptosis. (A) Western blotting results of NLRP3, ASC, cleaved caspase-1, cleaved caspase-8, GSDMD, and mature IL-1 $\beta$ in the cortex of the perihematomal regions at 3 days post-ICH. (B) Quantitative analysis of target proteins. Data are expressed as the mean \pm SEM $(\mathrm{n}=3)\left({ }^{* *}, \mathrm{P}<0.01 ;{ }^{* * *}, \mathrm{P}<0.001\right)$. Abbreviations: OM-MSCs, olfactory mucosa mesenchymal stem cells; ICH, intracerebral hemorrhage; NLRP3, nod-like receptor family protein 3; ASC, apoptosis-associated speck-like proteins containing a caspase recruitment domain; GSDMD, Gasdermin D; SEM, standard error of mean.

hypoxia-preconditioned OM-MSCs attenuated microglial pyroptosis in the ICH model.

\section{Discussion}

\section{Strategies enhancing therapeutic efficiency of MSCs in ICH}

MSCs have emerged as a promising therapeutic tool in ICH. Although naïve MSCs are still the most commonly used approach in ICH, research is being conducted to discover several methods to culture more functional MSCs, including pharmacological pre-conditioning (53), altering cell delivery approaches (54-56), genetic modification (57,58), physical method (59), and modification of culture conditions $(44,45)$. Hypoxic preconditioning is a kind of modification of culture conditions. In a general setting, MSCs are cultured in the normoxic niche $(\sim 21 \%)$. The ICH microenvironment is known to be hypoxic compared to normal brain tissues. Previous studies have demonstrated that hypoxiapreconditioned bone marrow-derived MSCs (44) and hypoxia-preconditioned neural stem cells (45) promote neurological functional recovery after ICH. The possible mechanism involves the increased neurogenesis, paracrine action, and increased cell survival in vivo. However, whether hypoxia-preconditioned MSCs decrease inflammation in ICH remains unclear. The present study investigated the hypothesis that hypoxic preconditioning can improve the therapeutic effects of OM-MSCs in mice subjected to ICH. We evaluated the neurobehavioral function and neuronal apoptosis, and the results showed that compared with normoxic OM-MSC transplantation, hypoxic preconditioning improved neurological deficit symptoms and alleviated neuronal apoptosis.

\section{Microglial pyroptosis and ICH pathologies}

Microglia, the resident immune cells of the central 
nervous system, mediates the inflammatory response after ICH. Microglia have been shown to play dual roles in secondary cerebral injury after ICH (5). On the one hand, microglia activation activates pro-inflammatory cytokines to promote inflammation, further exacerbating tissue injury. On the other hand, some microglial processes may be beneficial, such as clearing up debris and producing anti-inflammatory factors. Pyroptosis, or programmed necrosis, is characterized by the release of large numbers of pro-inflammatory cytokines. After a stroke, microglial pyroptosis is activated in the brain tissues, which could be mediated by NLRC4 $(27,60)$ and NLRP3 $(30,61,62)$. NLRP3 knockdown reduces brain edema and improves neurological functions from 24 to 72 hours following ICH (19). Some evidence has indicated that the NLRP3 inflammasome-mediated neuron pyroptosis is partially alleviated by promoting microglial polarization into the M2 phenotype, suggesting that microglial polarization could affect neuron pyroptosis (63).

Meanwhile, inhibiting NLRP3 inflammation activation could promote the shift of microglia from the M1 to the M2 phenotype after ICH (13). Most studies researching microglia polarization and microglia pyroptosis have only demonstrated that drugs or molecules could inhibit microglia pyroptosis and promote microglial polarization to M2 phenotype $(61,64,65)$. The precise relationship between microglia polarization and microglia pyroptosis after ICH remains unknown, and the potential vicious feedback cycle warrants further investigation.

The molecules regulating microglial pyroptosis include triggering receptor expressed on myeloid cells-1 (TREM-1) $(28,30)$, CD73 (66), native alpha-7 nicotinic subunit and its negative dominant duplicate (CHRFAM7A) (61), toll-like receptor 4 (67), LncRNA-Fendrr (60), LncRNA H19 (68), miRNA-27a (69), and nuclear factor erythroid 2-related factor 2 (70). Suppressing microglial pyroptosis could alleviate consequential neuroinflammation, early neuronal loss, brain-blood barrier breakdown and ultimately improve neurological outcomes after stroke. Thus, downregulating microglial pyroptosis might serve as a potential therapeutic strategy for ICH.

\section{Effects of MSCs on microglial pyroptosis post-ICH}

MSCs are well known to have anti-inflammatory effects on ICH (36,71-73). MSCs could attenuate the inflammatory response after hemorrhagic stroke by inhibiting microglial activation and microglial M1 polarization $(38,74)$. However, to our knowledge, no study has revealed the effects of MSCs on microglial pyroptosis post-ICH. In the present study, we discovered that hypoxic preconditioning enhanced the antiinflammatory effects of OM-MSCs, which may be related to the suppression of microglial pyroptosis in post-ICH brain tissues of the cerebral cortex. Hypoxia-preconditioned OMMSC could downregulate the expression of pyroptosisassociated proteins in perihematomal brain tissues, decrease the expression of microglial NLRP3 and caspase-1, and reduce membrane pores on microglia post-ICH. Although we suggested that hypoxic OM-MSCs inhibited microglial pyroptosis in ICH, the specific mechanism of the inflammatory response of microglia regulated by hypoxic OM-MSCs after ICH injury is still unclear. Previously, we demonstrated that OM-MSCs exerts neuroprotective effects on cerebral ischemia/reperfusion injury $(47,48,50)$. We also revealed that hypoxia-preconditioned OM-MSCs inhibit pyroptotic death of microglial cells by activating the hypoxia inducible factor$1 \alpha(\mathrm{HIF}-1 \alpha)$ in vitro model of ischemic stroke (49). However, the mechanism of OM-MSCs regulating microglial pyroptosis after stroke requires further study.

Accumulated evidence supports the notion that paracrine effects of transplanted MSCs are likely the major approach to alleviate neuroinflammation. MSC-derived extracellular vesicles are thought to carry functional molecules to neuron or glial cells and exhibit potent immunomodulatory and immunosuppressive effects on stroke. MSC-derived exosomes could ameliorate NLRP3 inflammasomemediated pyroptosis and neuroinflammation after stroke (63). Some bioactive molecules secreted by MSCs, such as the TNF- $\alpha$-stimulated gene 6 protein $(75,76)$ and miRNA-126 (77), play important anti-neuroinflammatory roles in stroke. Previously, we reviewed the signaling pathways involved in the immunosuppressive effects of MSCs (78). We will investigate the possible mechanism of hypoxic OM-MSCs regulation of microglial pyroptosis in $\mathrm{ICH}$ in future research.

\section{Limitations}

There were several limitations in this study that should be noted. Firstly, the use of a single transplantation time point, a single administration route, and a fixed cell dosing regimen. Secondly, only the mNSS and rotarod test were applied, which might not be optimal for long-term examination of behavioral function recovery. Thirdly, we only evaluated the immunofluorescence of NLRP3 and cleaved caspase- 1 in microglia but did not investigate the 

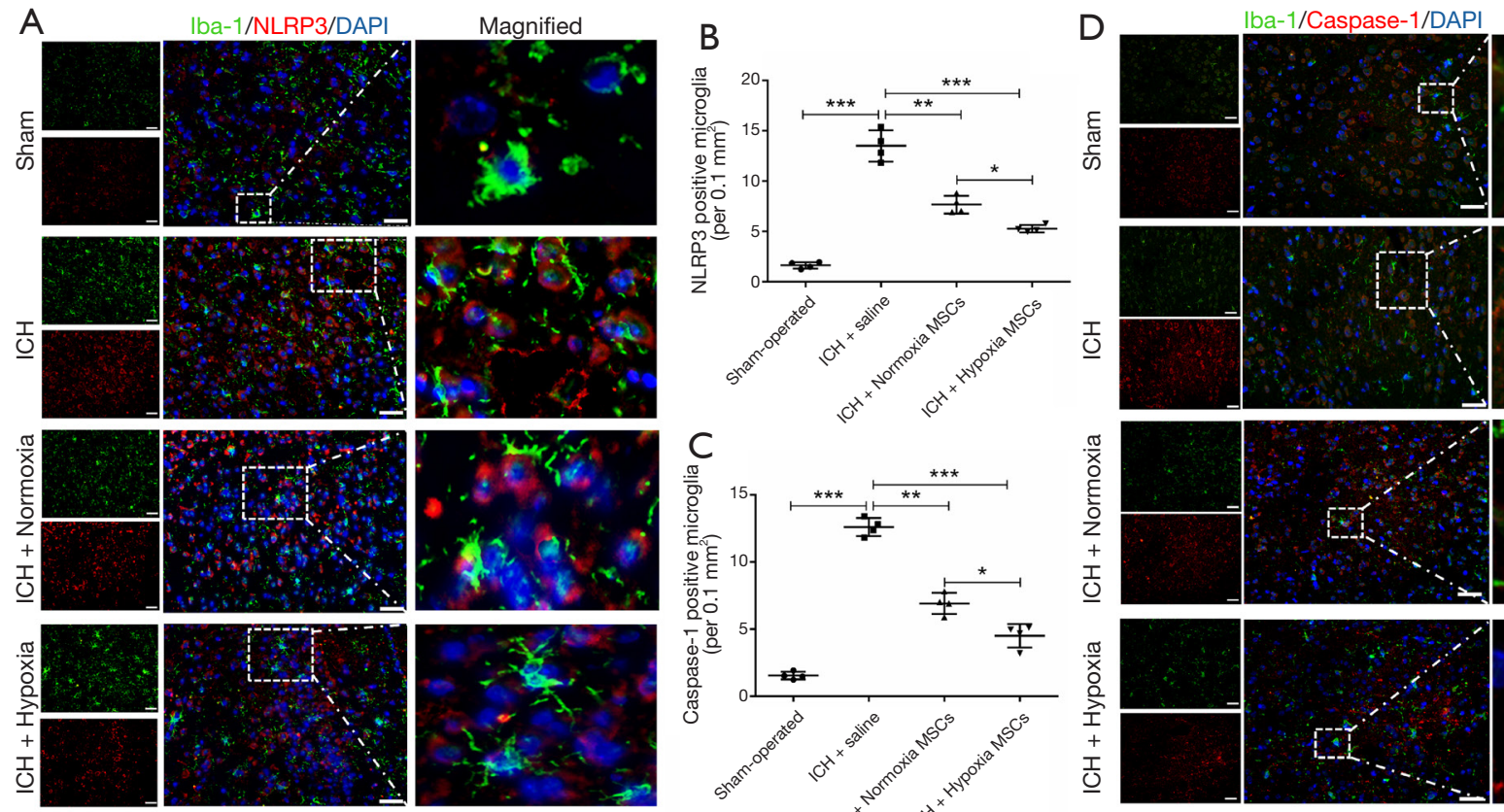

Magnified
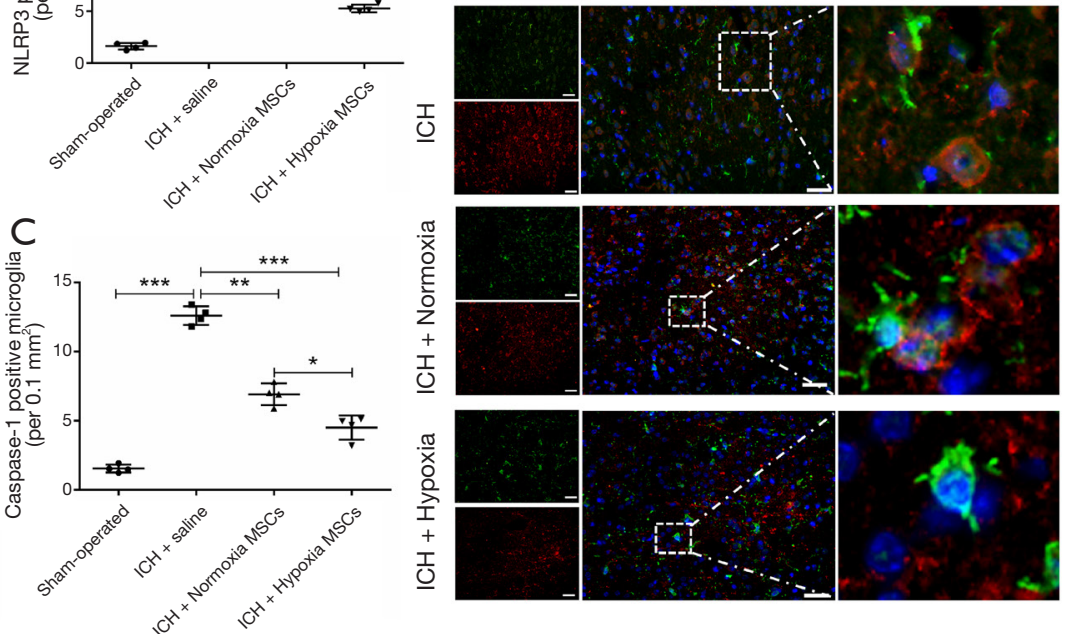

$\mathrm{E}$

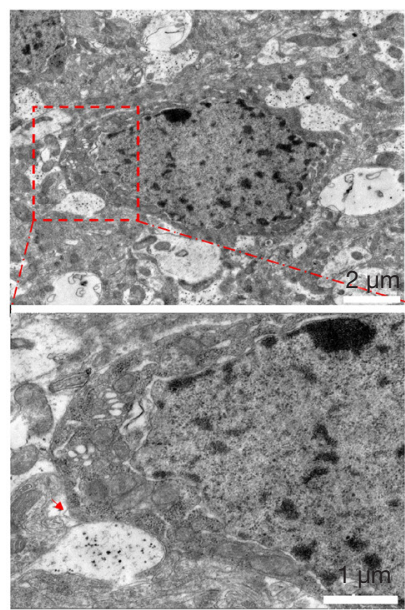

Sham

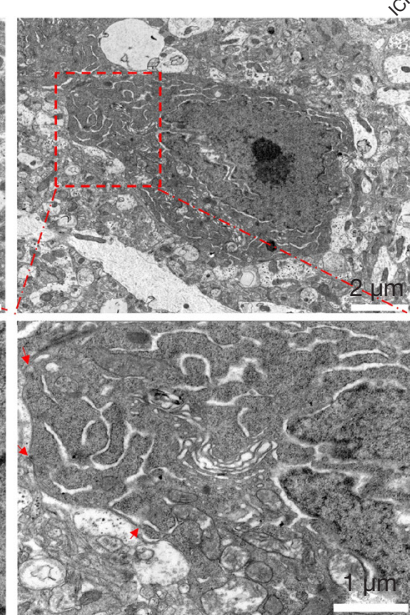

$\mathrm{ICH}$

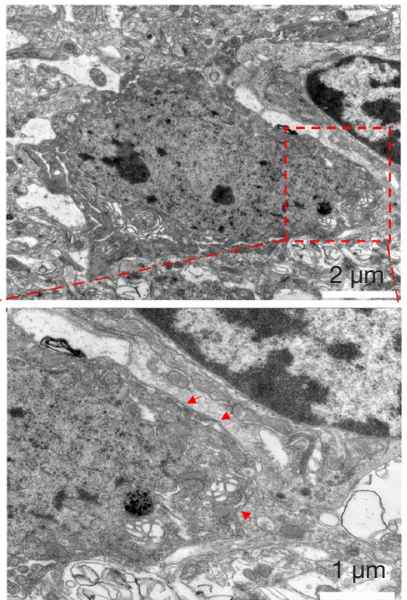

$\mathrm{ICH}+$ Normoxia
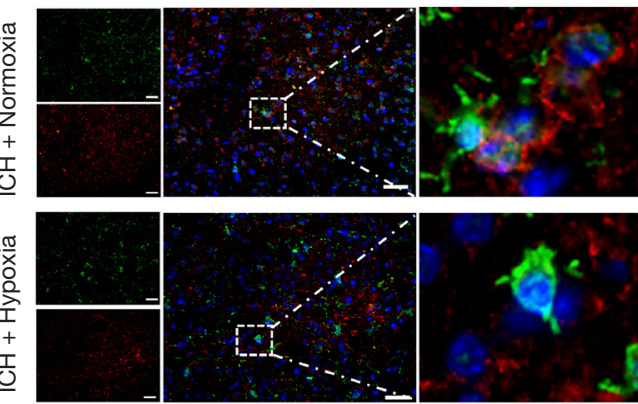

Figure 5 Hypoxia-preconditioned OM-MSCs attenuated microglial pyroptosis following ICH. (A,B) Iba1/NLRP3 double immunofluorescence staining in the cerebral cortex of perihematomal brain tissue, and quantitative analysis of NLRP3-positive microglia. The density and intensity of Iba1/NLRP3 double positive cells were increased at 3 days after ICH, which was decreased by OM-MSC administration, particularly hypoxic OM-MSCs. The scale bar is $40 \mu \mathrm{m}$. (C,D) Iba1/caspase-1 double immunofluorescence staining in the cerebral cortex of perihematomal brain tissue, and quantitative analysis of caspase-1-positive microglia. The density of Iba1/caspase-1 double positive cells was increased after ICH, while OM-MSC treatment decreased the positive signals in the perihematomal area. The scale bar is $40 \mu \mathrm{m}$. Data were expressed as the mean $\pm \operatorname{SEM}(\mathrm{n}=4)\left({ }^{*}, \mathrm{P}<0.05 ;{ }^{* *}, \mathrm{P}<0.01\right.$; $\left.{ }^{* * *}, \mathrm{P}<0.001\right)$. (E) Representative transmission electron microscopy pictures of microglia in the cortex of the perihematomal region. Amplified images of the cytomembrane are labeled using red boxes. Pores on the neuronal membrane are pointed out with red arrows. Insets show a higher magnification view. (n=3). OM-MSCs, olfactory mucosa mesenchymal stem cells; ICH, intracerebral hemorrhage; SEM, standard error of mean. 
pyroptosis of other cells (such as neurons or astrocytes).

\section{Conclusions}

This study demonstrated that hypoxic preconditioning enhanced the therapeutic efficiency of OM-MSCs in the ICH model, and hypoxic OM-MSCs significantly ameliorated microglial pyroptosis post-ICH.

\section{Acknowledgments}

Funding: This work was supported by grants from the National Natural Science Foundation of China (No. 81974213; No. 81801188) and the National Science Foundation of Hunan Province, China (No. 2019JJ40421).

\section{Footnotes}

Reporting Checklist: The authors have completed the ARRIVE reporting checklist. Available at https://dx.doi. org/10.21037/atm-21-2590

Data Sharing Statement: Available at https://dx.doi. org/10.21037/atm-21-2590

Conflicts of Interest: All authors have completed the ICMJE uniform disclosure form (available at https://dx.doi. org/10.21037/atm-21-2590). The authors have no conflicts of interest to declare.

Ethical Statement: The authors are accountable for all aspects of the work in ensuring that questions related to the accuracy or integrity of any part of the work are appropriately investigated and resolved. Animal Experiments were performed under a project license (No. 2020-110 164) granted by the institutional ethics board of Hunan Normal University, in compliance with the National Institutes of Health Guide for Care and Use of Laboratory Animals. A protocol was prepared before the study without registration. Studies involving human participants were reviewed and approved by the ethics committee of the Second Affiliated Hospital of Hunan Normal University (Changsha, China) (No. 2009163009) and conducted in accordance with the Declaration of Helsinki (as revised in 2013). The patients/ participants provided their written informed consent to participate in this study.

Open Access Statement: This is an Open Access article distributed in accordance with the Creative Commons Attribution-NonCommercial-NoDerivs 4.0 International License (CC BY-NC-ND 4.0), which permits the noncommercial replication and distribution of the article with the strict proviso that no changes or edits are made and the original work is properly cited (including links to both the formal publication through the relevant DOI and the license). See: https://creativecommons.org/licenses/by-nc-nd/4.0/.

\section{References}

1. Sacco S, Marini C, Toni D, et al. Incidence and 10-year survival of intracerebral hemorrhage in a population-based registry. Stroke 2009;40:394-9.

2. Zhu H, Wang Z, Yu J, et al. Role and mechanisms of cytokines in the secondary brain injury after intracerebral hemorrhage. Prog Neurobiol 2019;178:101610.

3. Wu H, Zhang Z, Li Y, et al. Time course of upregulation of inflammatory mediators in the hemorrhagic brain in rats: correlation with brain edema. Neurochem Int 2010;57:248-53.

4. Bonsack F 4th, Alleyne CH Jr, Sukumari-Ramesh S. Augmented expression of TSPO after intracerebral hemorrhage: a role in inflammation? J Neuroinflammation 2016;13:151.

5. Dudvarski Stankovic N, Teodorczyk M, Ploen R, et al. Microglia-blood vessel interactions: a double-edged sword in brain pathologies. Acta Neuropathol 2016;131:347-63.

6. Hu X, Liou AK, Leak RK, et al. Neurobiology of microglial action in CNS injuries: receptor-mediated signaling mechanisms and functional roles. Prog Neurobiol 2014;119-120:60-84.

7. Zhang Z, Zhang Z, Lu H, et al. Microglial Polarization and Inflammatory Mediators After Intracerebral Hemorrhage. Mol Neurobiol 2017;54:1874-86.

8. Lan X, Han X, Li Q, et al. Modulators of microglial activation and polarization after intracerebral haemorrhage. Nat Rev Neurol 2017;13:420-33.

9. Zi L, Zhou W, Xu J, et al. Rosuvastatin Nanomicelles Target Neuroinflammation and Improve Neurological Deficit in a Mouse Model of Intracerebral Hemorrhage. Int J Nanomedicine 2021;16:2933-47.

10. Yang $\mathrm{H}, \mathrm{Ni} W$, Wei $\mathrm{P}$, et al. HDAC inhibition reduces white matter injury after intracerebral hemorrhage. $\mathrm{J}$ Cereb Blood Flow Metab 2021;41:958-74.

11. Tschoe C, Bushnell CD, Duncan PW, et al. Neuroinflammation after Intracerebral Hemorrhage and Potential Therapeutic Targets. J Stroke 2020;22:29-46. 
12. Fu X, Zeng H, Zhao J, et al. Inhibition of Dectin-1 Ameliorates Neuroinflammation by Regulating Microglia/ Macrophage Phenotype After Intracerebral Hemorrhage in Mice. Transl Stroke Res 2021. doi: 10.1007/S12975021-00889-2

13. Chen W, Guo C, Huang S, et al. MitoQ attenuates brain damage by polarizing microglia towards the M2 phenotype through inhibition of the NLRP3 inflammasome after ICH. Pharmacol Res 2020;161:105122.

14. Lin L, Yihao T, Zhou F, et al. Inflammatory Regulation by Driving Microglial M2 Polarization: Neuroprotective Effects of Cannabinoid Receptor-2 Activation in Intracerebral Hemorrhage. Front Immunol 2017;8:112.

15. He Y, Gao Y, Zhang Q, et al. IL-4 Switches Microglia/ macrophage M1/M2 Polarization and Alleviates Neurological Damage by Modulating the JAK1/STAT6 Pathway Following ICH. Neuroscience 2020;437:161-71.

16. Zhao JL, Chen YJ, Yu J, et al. ISO-alpha-acids improve the hematoma resolution and prevent peri-hematoma inflammations by transforming microglia via PPARgammaCD36 axis in ICH rats. Int Immunopharmacol 2020;83:106396.

17. Wu J, Sun L, Li H, et al. Roles of programmed death protein 1/programmed death-ligand 1 in secondary brain injury after intracerebral hemorrhage in rats: selective modulation of microglia polarization to anti-inflammatory phenotype. J Neuroinflammation 2017;14:36.

18. Bergsbaken T, Fink SL, Cookson BT. Pyroptosis: host cell death and inflammation. Nat Rev Microbiol 2009;7:99-109.

19. Ma Q, Chen S, Hu Q, et al. NLRP3 inflammasome contributes to inflammation after intracerebral hemorrhage. Ann Neurol 2014;75:209-19.

20. Xiao L, Zheng H, Li J, et al. Neuroinflammation Mediated by NLRP3 Inflammasome After Intracerebral Hemorrhage and Potential Therapeutic Targets. Mol Neurobiol 2020;57:5130-49.

21. Shi J, Zhao Y, Wang K, et al. Cleavage of GSDMD by inflammatory caspases determines pyroptotic cell death. Nature 2015;526:660-5.

22. Fantuzzi G, Dinarello CA. Interleukin-18 and interleukin-1 beta: two cytokine substrates for ICE (caspase-1). J Clin Immunol 1999;19:1-11.

23. Ding J, Wang K, Liu W, et al. Pore-forming activity and structural autoinhibition of the gasdermin family. Nature 2016;535:111-6.

24. Sekerdag E, Solaroglu I, Gursoy-Ozdemir Y. Cell Death Mechanisms in Stroke and Novel Molecular and
Cellular Treatment Options. Curr Neuropharmacol 2018;16:1396-415.

25. Sun R, Peng M, Xu P, et al. Low-density lipoprotein receptor (LDLR) regulates NLRP3-mediated neuronal pyroptosis following cerebral ischemia/reperfusion injury. J Neuroinflammation 2020;17:330.

26. Feng L, Chen Y, Ding R, et al. P2X7R blockade prevents NLRP3 inflammasome activation and brain injury in a rat model of intracerebral hemorrhage: involvement of peroxynitrite. J Neuroinflammation 2015;12:190.

27. Poh L, Kang SW, Baik SH, et al. Evidence that NLRC4 inflammasome mediates apoptotic and pyroptotic microglial death following ischemic stroke. Brain Behav Immun 2019;75:34-47.

28. Xu P, Zhang X, Liu Q, et al. Microglial TREM-1 receptor mediates neuroinflammatory injury via interaction with SYK in experimental ischemic stroke. Cell Death Dis 2019;10:555.

29. Wang K, Ru J, Zhang H, et al. Melatonin Enhances the Therapeutic Effect of Plasma Exosomes Against Cerebral Ischemia-Induced Pyroptosis Through the TLR4/NF- $\kappa \mathrm{B}$ Pathway. Front Neurosci 2020;14:848.

30. Xu P, Hong Y, Xie Y, et al. TREM-1 Exacerbates Neuroinflammatory Injury via NLRP3 InflammasomeMediated Pyroptosis in Experimental Subarachnoid Hemorrhage. Transl Stroke Res 2021;12:643-59.

31. Chang Y, Zhu J, Wang D, et al. NLRP3 inflammasomemediated microglial pyroptosis is critically involved in the development of post-cardiac arrest brain injury. J Neuroinflammation 2020;17:219.

32. Vinukonda G, Liao Y, Hu F, et al. Human Cord BloodDerived Unrestricted Somatic Stem Cell Infusion Improves Neurobehavioral Outcome in a Rabbit Model of Intraventricular Hemorrhage. Stem Cells Transl Med 2019;8:1157-69.

33. Feng M, Zhu H, Zhu Z, et al. Serial 18F-FDG PET demonstrates benefit of human mesenchymal stem cells in treatment of intracerebral hematoma: a translational study in a primate model. J Nucl Med 2011;52:90-7.

34. Chen J, Tang YX, Liu YM, et al. Transplantation of adipose-derived stem cells is associated with neural differentiation and functional improvement in a rat model of intracerebral hemorrhage. CNS Neurosci Ther 2012;18:847-54.

35. Zhang Y, Deng H, Hu Y, et al. Adipose-derived mesenchymal stem cells stereotactic transplantation alleviate brain edema from intracerebral hemorrhage. J Cell Biochem 2019;120:14372-82. 
36. Chen M, Li X, Zhang X, et al. The inhibitory effect of mesenchymal stem cell on blood-brain barrier disruption following intracerebral hemorrhage in rats: contribution of TSG-6. J Neuroinflammation 2015;12:61.

37. Lee HS, Kim KS, Lim HS, et al. Priming Wharton's jelly-derived mesenchymal stromal/stem cells with ROCK inhibitor improves recovery in an intracerebral hemorrhage model. J Cell Biochem 2015;116:310-9.

38. Kim S, Kim YE, Hong S, et al. Reactive microglia and astrocytes in neonatal intraventricular hemorrhage model are blocked by mesenchymal stem cells. Glia 2020;68:178-92.

39. Hu Y, Liu N, Zhang P, et al. Preclinical Studies of Stem Cell Transplantation in Intracerebral Hemorrhage: a Systemic Review and Meta-Analysis. Mol Neurobiol 2016;53:5269-77.

40. Luo Z, Wu F, Xue E, et al. Hypoxia preconditioning promotes bone marrow mesenchymal stem cells survival by inducing HIF-1 $\alpha$ in injured neuronal cells derived exosomes culture system. Cell Death Dis 2019;10:134.

41. Chang HH, Hsu SP, Chien CT. Intrarenal Transplantation of Hypoxic Preconditioned Mesenchymal Stem Cells Improves Glomerulonephritis through Anti-Oxidation, Anti-ER Stress, Anti-Inflammation, Anti-Apoptosis, and Anti-Autophagy. Antioxidants (Basel) 2019;9:2.

42. Jiang RH, Wu CJ, Xu XQ, et al. Hypoxic conditioned medium derived from bone marrow mesenchymal stromal cells protects against ischemic stroke in rats. J Cell Physiol 2019;234:1354-68.

43. Zhou P, Tan YZ, Wang HJ, et al. Hypoxic preconditioning-induced autophagy enhances survival of engrafted endothelial progenitor cells in ischaemic limb. J Cell Mol Med 2017;21:2452-64.

44. Sun J, Wei ZZ, Gu X, et al. Intranasal delivery of hypoxiapreconditioned bone marrow-derived mesenchymal stem cells enhanced regenerative effects after intracerebral hemorrhagic stroke in mice. Exp Neurol 2015;272:78-87.

45. Wakai T, Narasimhan P, Sakata H, et al. Hypoxic preconditioning enhances neural stem cell transplantation therapy after intracerebral hemorrhage in mice. J Cereb Blood Flow Metab 2016;36:2134-45.

46. Nivet E, Vignes M, Girard SD, et al. Engraftment of human nasal olfactory stem cells restores neuroplasticity in mice with hippocampal lesions. J Clin Invest 2011;121:2808-20.

47. Liu J, Huang Y, He J, et al. Olfactory Mucosa Mesenchymal Stem Cells Ameliorate Cerebral Ischemic/ Reperfusion Injury Through Modulation of UBIAD1
Expression. Front Cell Neurosci 2020;14:580206.

48. He J, Liu J, Huang Y, et al. Olfactory Mucosa

Mesenchymal Stem Cells Alleviate Cerebral Ischemia/ Reperfusion Injury Via Golgi Apparatus Secretory Pathway Ca2+-ATPase Isoform1. Front Cell Dev Biol 2020;8:586541.

49. Huang Y, Tan F, Zhuo Y, et al. Hypoxia-preconditioned olfactory mucosa mesenchymal stem cells abolish cerebral ischemia/reperfusion-induced pyroptosis and apoptotic death of microglial cells by activating HIF-1 $\alpha$. Aging (Albany NY) 2020;12:10931-50.

50. Zhuo $\mathrm{Y}$, Chen $\mathrm{W}, \mathrm{Li} \mathrm{W}$, et al. Ischemic-hypoxic preconditioning enhances the mitochondrial function recovery of transplanted olfactory mucosa mesenchymal stem cells via miR-181a signaling in ischemic stroke. Aging (Albany NY) 2021;13:11234-56.

51. Bederson JB, Pitts LH, Tsuji M, et al. Rat middle cerebral artery occlusion: evaluation of the model and development of a neurologic examination. Stroke 1986;17:472-6.

52. He J, Huang Y, Liu J, et al. Hypoxic conditioned promotes the proliferation of human olfactory mucosa mesenchymal stem cells and relevant lncRNA and mRNA analysis. Life Sci 2021;265:118861.

53. Nizamudeen ZA, Chakrabarti L, Sottile V. Exposure to the ROCK inhibitor fasudil promotes gliogenesis of neural stem cells in vitro. Stem Cell Res 2018;28:75-86.

54. Liu Y, Hsu YH, Huang AP, et al. Semi-Interpenetrating Polymer Network of Hyaluronan and Chitosan SelfHealing Hydrogels for Central Nervous System Repair. ACS Appl Mater Interfaces 2020;12:40108-20.

55. Chung CY, Yang JT, Kuo YC. Polybutylcyanoacrylate nanoparticles for delivering hormone response elementconjugated neurotrophin-3 to the brain of intracerebral hemorrhagic rats. Biomaterials 2013;34:9717-27.

56. Gong Y, Wang Y, Qu Q, et al. Nanoparticle encapsulated core-shell hydrogel for on-site BMSCs delivery protects from iron overload and enhances functional recovery. J Control Release 2020;320:381-91.

57. Ko HR, Ahn SY, Chang YS, et al. Human UCB-MSCs treatment upon intraventricular hemorrhage contributes to attenuate hippocampal neuron loss and circuit damage through BDNF-CREB signaling. Stem Cell Res Ther 2018;9:326.

58. Wakai T, Sakata H, Narasimhan P, et al. Transplantation of neural stem cells that overexpress SOD1 enhances amelioration of intracerebral hemorrhage in mice. J Cereb Blood Flow Metab 2014;34:441-9.

59. Cui M, Ge H, Zeng H, et al. Repetitive Transcranial 
Magnetic Stimulation Promotes Neural Stem Cell Proliferation and Differentiation after Intracerebral Hemorrhage in Mice. Cell Transplant 2019;28:568-84.

60. Wang LQ, Zheng YY, Zhou HJ, et al. LncRNA-Fendrr protects against the ubiquitination and degradation of NLRC4 protein through HERC2 to regulate the pyroptosis of microglia. Mol Med 2021;27:39.

61. Cao X, Wang Y, Gao L. CHRFAM7A Overexpression Attenuates Cerebral Ischemia-Reperfusion Injury via Inhibiting Microglia Pyroptosis Mediated by the NLRP3/ Caspase-1 pathway. Inflammation 2021;44:1023-34.

62. Li X, Wang T, Zhang D, et al. Andrographolide ameliorates intracerebral hemorrhage induced secondary brain injury by inhibiting neuroinflammation induction. Neuropharmacology 2018;141:305-15.

63. Liu X, Zhang M, Liu H, et al. Bone marrow mesenchymal stem cell-derived exosomes attenuate cerebral ischemiareperfusion injury-induced neuroinflammation and pyroptosis by modulating microglia M1/M2 phenotypes. Exp Neurol 2021;341:113700.

64. Ma DC, Zhang NN, Zhang YN, et al. Salvianolic Acids for Injection alleviates cerebral ischemia/reperfusion injury by switching M1/M2 phenotypes and inhibiting NLRP3 inflammasome/pyroptosis axis in microglia in vivo and in vitro. J Ethnopharmacol 2021;270:113776.

65. Lin X, Ye H, Siaw-Debrah F, et al. AC-YVAD-CMK Inhibits Pyroptosis and Improves Functional Outcome after Intracerebral Hemorrhage. Biomed Res Int 2018;2018:3706047.

66. Xu S, Wang J, Zhong J, et al. CD73 alleviates GSDMDmediated microglia pyroptosis in spinal cord injury through PI3K/AKT/Foxo1 signaling. Clin Transl Med 2021;11:e269.

67. Xu S, Wang J, Jiang J, et al. TLR4 promotes microglial pyroptosis via lncRNA-F630028O10Rik by activating PI3K/AKT pathway after spinal cord injury. Cell Death Dis 2020;11:693.

68. Wan $\mathrm{P}, \mathrm{Su} W$, Zhang Y, et al. LncRNA H19 initiates microglial pyroptosis and neuronal death in retinal ischemia/reperfusion injury. Cell Death Differ 2020;27:176-91.

69. Li Y, Song W, Tong Y, et al. Isoliquiritin ameliorates depression by suppressing NLRP3-mediated pyroptosis via miRNA-27a/SYK/NF- $\kappa$ B axis. J Neuroinflammation 2021;18:1.

70. Arioz BI, Tastan B, Tarakcioglu E, et al. Melatonin Attenuates LPS-Induced Acute Depressive-Like Behaviors and Microglial NLRP3 Inflammasome Activation Through the SIRT1/Nrf2 Pathway. Front Immunol 2019;10:1511.

71. Kim K, Park HW, Moon HE, et al. The Effect of Human Umbilical Cord Blood-Derived Mesenchymal Stem Cells in a Collagenase-Induced Intracerebral Hemorrhage Rat Model. Exp Neurobiol 2015;24:146-55.

72. Liao W, Zhong J, Yu J, et al. Therapeutic benefit of human umbilical cord derived mesenchymal stromal cells in intracerebral hemorrhage rat: implications of antiinflammation and angiogenesis. Cell Physiol Biochem 2009;24:307-16.

73. Zhou H, Zhang H, Yan Z, et al. Transplantation of human amniotic mesenchymal stem cells promotes neurological recovery in an intracerebral hemorrhage rat model. Biochem Biophys Res Commun 2016;475:202-8.

74. Duan S, Wang F, Cao J, et al. Exosomes Derived from MicroRNA-146a-5p-Enriched Bone Marrow Mesenchymal Stem Cells Alleviate Intracerebral Hemorrhage by Inhibiting Neuronal Apoptosis and Microglial M1 Polarization. Drug Des Devel Ther 2020;14:3143-58.

75. Yang $\mathrm{H}, \mathrm{Wu} \mathrm{L}$, Deng $\mathrm{H}$, et al. Anti-inflammatory protein TSG-6 secreted by bone marrow mesenchymal stem cells attenuates neuropathic pain by inhibiting the TLR2/ MyD88/NF- $\kappa$ B signaling pathway in spinal microglia. J Neuroinflammation 2020;17:154.

76. Jha KA, Pentecost M, Lenin R, et al. TSG-6 in conditioned media from adipose mesenchymal stem cells protects against visual deficits in mild traumatic brain injury model through neurovascular modulation. Stem Cell Res Ther 2019;10:318.

77. Geng W, Tang H, Luo S, et al. Exosomes from miRNA126-modified ADSCs promotes functional recovery after stroke in rats by improving neurogenesis and suppressing microglia activation. Am J Transl Res 2019;11:780-92.

78. He J, Liu J, Huang Y, et al. Oxidative Stress, Inflammation, and Autophagy: Potential Targets of Mesenchymal Stem Cells-Based Therapies in Ischemic Stroke. Front Neurosci 2021;15:641157.

(English Language Editors: A. Kassem and J. Chapnick)

Cite this article as: Liu J, He J, Huang Y, Ge L, Xiao H, Zeng L, Jiang Z, Lu M, Hu Z. Hypoxia-preconditioned mesenchymal stem cells attenuate microglial pyroptosis after intracerebral hemorrhage. Ann Transl Med 2021;9(17):1362. doi: 10.21037/atm-21-2590 